\title{
Cytomegalovirus colitis in immunocompetent individual
}

\author{
Husayn Al Mahdy
}

\begin{abstract}
A woman with peripheral vascular disease developed cytomegalovirus colitis following repair of abdominal aortic aneurysm. Cytomegalovirus colitis developing in an immunocompetent individual may be caused by a breach in the integrity of the mucosal lining of the colon from various causes and should alert the clinician to explore these causes in order to provide effective care.

(f Clin Pathol 1998;51:475-476)
\end{abstract}

Keywords: cytomegalovirus colitis; immune competence

\section{Case report}

A 72 year old woman with long standing atrial fibrillation from ischaemic heart disease, hypertension, and intermittent claudication in the left leg presented with back pain caused by an abdominal aortic aneurysm. There was no other significant past medical history. She was taking warfarin for chronic atrial fibrillation, perindopril for hypertension, and antacids intermittently for indigestion. An echocardiogram showed mild left ventricular hypertrophy with an ejection fraction of approximately $70 \%$. Computed tomography (CT) of the abdomen showed an aortic aneurysm with thrombus and a calcified wall, with the bulk of the aneurysm below the renal arteries and extending to the bifurcation. The iliac, splenic, and parts of the femoral arteries were calcified. No other pathology was noted. The chest $x$ ray

Accepted for publication 12 March 1998

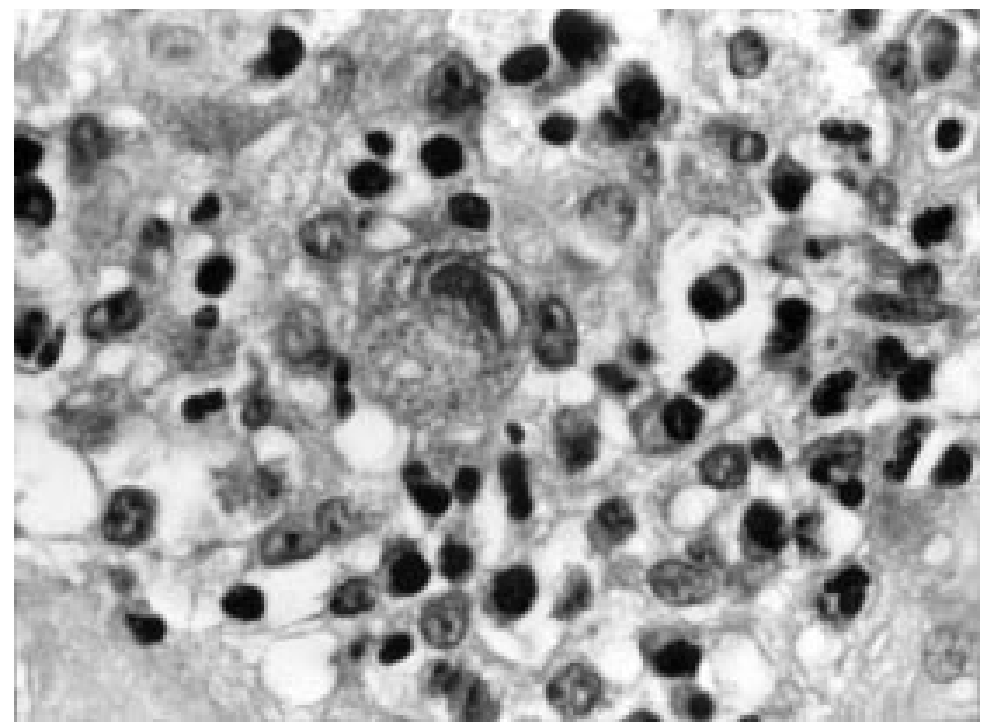

Figure 1 Cell infected by cytomegalovirus with large intranuclear inclusion and perinuclear halo. Smaller intracytoplasmic inclusions are also present. Haematoxylin and eosin, $\times 75$. showed clear lung fields. The blood count, electrolytes, and liver function tests were normal and urea was mildly raised at 7.5 $\mathrm{mmol} /$ litre.

The abdominal aortic aneurysm was repaired but in the immediate postoperative period the patient became jaundiced with deranged liver function tests. This was thought to be a result of perioperative hypoperfusion of the liver. A few days later she developed acute pancreatitis, and abdominal CT showed an oedematous pancreas compressing the stomach and duodenum, as well as nodular thickening of the rectal wall. She was treated conservatively and improved.

Her general condition was improving clinically, but on the 12th postoperative day she developed diarrhoea. Stool microscopy revealed moderate numbers of leucocytes and a few red cells, but culture failed to grow bacterial pathogens. Clostridium difficile toxin was not detected. Another stool specimen was examined a few days later but there were no new features and it was still negative for Clostridium difficile toxin. Serology to cytomegalovirus was IgM negative but IgG positive, suggesting past exposure to the virus. Antibodies to HIV-1 and HIV-2 were negative by enzyme linked immunosorbent assay.

The diarrhoea continued and on occasions was blood stained. The patient was also mildly pyrexial. Approximately five weeks later she underwent flexible sigmoidoscopy which showed a large rectal polyp with ulceration in the sigmoid colon. It was thought the features were those of inflammatory bowel disease and she was treated empirically with salazopyrine and metronidazole. Histology of sigmoid biopsies showed ulceration with acute inflammatory debris in the granulation tissue. Some of the specimens showed deep ulceration involving the submucosa, with fibrin thrombi in capillaries suggesting ischaemia. Cells with inclusion bodies and immunoperoxidase staining were identified, confirming the presence of cytomegalovirus (fig 1). The features were thus those of cytomegalovirus colitis superimposed on ischaemia. Treatment with salazopyrine and metronidazole was stopped when the findings of the biopsies were known. The patient was treated conservatively and the diarrhoea resolved a month after onset.

Serum was taken for cytomegalovirus antibody titres and compared with the initial specimen taken at the time of diarrhoea onset. This initial specimen had an anti-cytomegalovirus IgG concentration of $53 \mathrm{UA} / \mathrm{ml}$, while the concentration in the serum collected a month later, at the time of resolution of diarrhoea, was 345 
$\mathrm{UA} / \mathrm{ml}$. After a further month the concentration was $207 \mathrm{UA} / \mathrm{ml}$. At about this time colonoscopy was undertaken to evaluate the state of the colonic mucosa. The mucosa in the sigmoid colon showed superficial ulceration. The rectal polyp that was seen during the initial flexible sigmoidoscopy was removed. Histological examination showed that it was a villous adenoma. Histology of the ulcerated sigmoid mucosa showed a markedly reduced inflammatory response, with no inclusion bodies in the cells. The patient was reviewed a few weeks later and there had been no recurrence of the diarrhoea.

\section{Discussion}

Cytomegalovirus colitis has been described in immunosuppressed individuals: in AIDS, ${ }^{12}$ with corticosteroid treatment, ${ }^{3}$ in severe illness, ${ }^{4}$ and patients who have had organ transplantation and are maintained on immune modulating drugs. ${ }^{5}$ There have also been case reports of patients with cytomegalovirus colitis associated with inflammatory bowel disease, ${ }^{67}$ where the predisposing factors may have been a combination of ulceration of the colon and corticosteroid treatment.

There have, however, been a few case reports of cytomegalovirus colitis in immunocompetent individuals apparently without predisposing factors. For example, a 39 year old pregnant woman developed self limiting acute ulcerative proctocolitis associated with primary cytomegalovirus infection which on follow up showed features of inflammatory bowel disease. ${ }^{8}$ It may be that this pregnant woman already had low grade proctitis without significant symptoms but cytomegalovirus invasion occurred in the presence of denuded rectal mucosa leading to exacerbation of "colitis," as in other reported cases with pre-existing ulcerative colitis. ${ }^{67}$ Similarly, two of three patients described by Surawicz and Myerson ${ }^{9}$ had had anal intercourse and developed self limiting cytomegalovirus colitis from the invasion of this organism following rectal mucosal damage. The third patient with seemingly no predisposing factors later developed Crohn's disease after the elimination of cytomegalovirus from colonic mucosa, and the diarrhoea responded to corticosteroids. It is possible that the initial process was Crohn's disease, which facilitated cytomegalovirus invasion of the mucosa and hence exacerbated the "colitis."

This case report of an immunocompetent woman developing cytomegalovirus colitis postoperatively supports the hypothesis that mucosal damage, in this case caused by ischaemia, is the common predisposing factor in the development of colitis from this organism in immunocompetent individuals. The second abdominal CT showed nodular thickening of the rectal wall suggesting ulceration and should have prompted immediate endoscopic evaluation when the diarrhoea developed.

The hypothesis of colonic mucosal damage from whatever cause as the essential factor predisposing to cytomegalovirus invasion needs to be subjected to further study.

Such studies need to investigate other factors that may be important in predicting outcome and hence in directing appropriate treatment of this disease in immunocompetent and possibly also immunosuppressed individuals.

1 Dieterich DT, Rahmin M. Cytomegalovirus colitis in AIDS: presentation in 44 patients and a review of the literature. $\mathcal{F}$ Acquir Immune Defic Syndrome 1991;4(suppl 1):S29-35.

2 Mentec H, Leport C, Leport J, et al. Cytomegalovirus colitis Mentec H, Leport C, Leport J, et al. Cytomegalovirus colitis
in HIV-1-infected patients: a prospective research in 55 in HIV-1-infected patients: a p
patients. AIDS 1994;8:461-67.

3 Aukrust P, Moum B, Farstad IN, et al. Fatal cytomegalovirus (CMV) colitis in a patient receiving low dose prednisolone therapy. Scand $\mathscr{f}$ Infect Dis 1991;23:495-9.

4 Jawad SH. CMV colitis in elderly. Postgrad Med $f$ 1992;68:484.

5 Onarato IM, Morens DM, Martone WJ, et al. Epidemiology of cytomegaloviral infections: recommendations for prevention and control. Rev Infect Dis 1985;7:479-91.

6 Kotanagi H, Fukuoka T, Shibata Y, et al. A case of toxic megacolon in ulcerative colitis associated with cytomegalomegacolon in ulcerative colitis associated with

7 Sidi S, Graham JH, Razvis A, et al. Cytomegalovirus infection of the colon associated with ulcerative colitis. Arch Surg 1979;114:857-9.

8 Diepersloot RJA, Kroes ACM, Visser W, et al. Acute ulcerative proctocolitis associated with primary cytomegalovirus infection. Arch Intern Med 1990;150:1749-51.

9 Surawicz CM, Myerson D. Self-limiting cytomegalovirus colitis in immunocompetent individuals. Gastroenterology 1988;94:194-9. 